\title{
'And lose the name of action'? Überlegungen zur Schüleraktivierung mit Drama und Film im Shakespeare-Unterricht
}

\author{
Christiane Lütge
}

\begin{abstract}
Zusammenfassung
Formen szenischen Interpretierens und die Beschäftigung mit unterschiedlichen Filmversionen eines Shakespeare-Dramas bieten zahlreiche Ansatzpunkte für Schüleraktivierung im Rahmen eines handlungsorientierten Englischunterrichts. Dieser Beitrag möchte Überlegungen einer performanceorientierten Methodik mit filmdidaktischen Aspekten zusammenführen und im Hinblick auf eine Konkretisierung im Shakespeare-Unterricht diskutieren. Der aufführungsorientierte Ansatz vermag ein Bewusstsein zu erzeugen für die Möglichkeiten der darstellerischen Umsetzung eines Dramas auf der Bühne und im Film. Szenische Darstellungen können für konkrete Fragen der Aufführungspraxis sensibilisieren und dabei die Eigentätigkeit und Selbstreflexivität der Lerner im Spannungsfeld zwischen Dramentext und Verfilmung fördern. Methodische Vorschläge einer schüleraktivierenden Annäherung an diese Thematik sollen helfen, die praktische Umsetzung im Shakespeare-Unterricht zu erleichtern.
\end{abstract}

\section{Einleitung}

Der Einsatz von Shakespeare-Verfilmungen im Englischunterricht erfreut sich in jüngster Zeit zunehmend größerer Beliebtheit, was nicht zuletzt auch auf die neueren Arbeiten von Baz Luhrmann und Kenneth Branagh zurückzuführen ist. Im DVD-Zeitalter ${ }^{1}$ ergeben sich dabei methodische Möglichkeiten, die im Rahmen vergleichender Analysen einen durch die Audiovision veränderten 'Blick' zurück auf den Dramentext ermöglichen. Allerdings wird das didaktische Potenzial an der Schnittstelle zwischen Drama und Film mit zahlreichen Möglichkeiten für schüleraktivierende Verfahren wie pantomimische Darstellungen

\footnotetext{
${ }^{1}$ Geradezu abenteuerlich muten die Preise und Bedingungen der Filmbeschaffung von Videokassetten an, die Hombitzer in ihrem Artikel zum Einsatz der Shakespeare-Verfilmung in der Sekundarstufe II beschreibt (vgl. Hombitzer 1986: 1011, Anmerkung 16). Wie sehr sich in 20 Jahren technische Veränderungen mit großem Einfluss auf didaktisch-methodische Handhabbarkeit ergeben haben, wird deutlich bei Thaler (2001).
} 
und szenische Improvisationen längst nicht immer genutzt. Die Gründe dafür sind vielfältig.

Noch allzu häufig wird das Medium Film für eine eher passive Haltung bei den Lernern verantwortlich gemacht. Wird die oft kritisierte, aber ebenso häufig praktizierte Filmvorführung im Anschluss an eine (Shakespeare-) Lektüre unreflektiert perpetuiert, bestätigt sich schnell das Bild einer eher distanzierten passiven Rezeption, die die Zuschauer zu bloßen Medienkonsumenten degradiert.

Zudem bewegen sich die Reaktionen der Lerner auf die Ankündigung einer Beschäftigung mit Shakespeare im Englischunterricht häufig auf einem Kontinuum zwischen respektvollem Interesse und letztlich ebenso respektvoller Ablehnung. Der tief verwurzelte Geniekult"(Volkmann 1997: 32) um Shakespeare ist häufig dazu angetan, eine distanzierte, zuweilen auch vorsichtig-abwartende Haltung zu schaffen. ${ }^{2}$

Bedeutet dies nun, dass der Einsatz von Shakespeare-Verfilmungen in besonderer Weise für eine Distanz zwischen Schülern und Lerngegenstand sorgt? Werden Schüler hier zu Passivität oder gar Resignation verleitet, so dass auf sie - wie auf Hamlet - im doppelten Wortsinn die Charakterisierung zutrifft: "... and lose the name of action"? ${ }^{3}$

Ich meine nein, denn die Gattung Drama und das Medium Film in Kombination bieten genügend Anknüpfungspunkte für schüleraktivierende Verfahren, die zu echter Involviertheit der Lerner führen und somit Passivität und Distanz wirkungsvoll begegnen können. Damit kann das Medium Film den Einsatz dramatischer Texte im Hinblick auf szenische Interpretationen im Englischunterricht wirkungsvoll unterstützen. Dies gilt in besonderer Weise für Shakespeare, dessen Dramen in einer Vielzahl unterschiedlicher Verfilmungen zur Verfügung stehen, was zu einer Fülle aufführungspraktischer Kontrastierungen einlädt. Im Folgenden möchte ich zunächst auf die Funktion von Filmen im Shakespeare-Unterricht eingehen, anschließend dramendidaktische Überlegungen zur Schüleraktivierung anstellen und danach methodische Möglichkeiten der schulischen Umsetzung diskutieren.

\section{Medialisierte Dramen: Verfilmungen im Shakespeare-Unterricht}

Wenn man im Shakespeare-Unterricht mehr will als das eingangs schon erwähnte, häufig kritisierte "Bonbonëiner der Dramenlektüre nachgeordneten Filmdarbietung, dann muss zunächst einmal das didaktische Potenzial von Shakespeare-Verfilmungen in den Blick genommen werden. Wie auch bei der Verfilmung anderer literarischer Werke kann eine Begründung natürlich

\footnotetext{
${ }^{2}$ Für deren Überwindung liegen allerdings durchaus zahlreiche Vorschläge im Bereich kreativer Ansätze vor. Vgl. hierzu z.B. Themenheft SShakespeare kreativ": Der fremdsprachliche Unterricht Englisch 36/56 (März 2002).

${ }^{3}$ Hamlet II, 1, 65.
} 
durchaus in der unterstellten motivationsfördernden Wirkung gesehen werden (vgl. Hombitzer 1986: 992), die vom Medium Film ausgehen kann. Im Rahmen neuerer filmdidaktischer Arbeiten wird jedoch gelegentlich die Klage laut, dass sich filmanalytische Verfahren und Formen handlungs- und prozessorientierter Arbeit mit Filmen diametral gegenüberstehen (vgl. Surkamp 2004). Tatsächlich lassen sich beide Ansätze aber gut miteinander verbinden und insbesondere an Shakespeare-Verfilmungen umsetzen.

Hombitzer betont, dass die Beobachtung des filmisches Codes die Aufmerksamkeit zuück lenkt auf die Ausdrucksmittel der Sprache, wodurch deutlich wird, dass Text und Verfilmung einander weder ersetzen können noch austauschbar sind (vgl. Hombitzer 1986: 1010). Die Erkenntnis, dass Shakespeare-Verfilmungen das Lesen nicht überflüssig machen, sondern dass sich die Begegnung mit einer Übertragung des Dramas in das Medium Film als erhellend erweist, wird durch die Kontrastierung mehrerer Verfilmungen noch verstärkt:

Die Auseinandersetzung mit mehreren Filmversionen des gleichen ShakespeareDramas, d.h. mit verschiedenen Interpretationen, mögen diese Filme vom selben oder von verschiedenen Regisseuren sein, zeigt, in welchem Umfang persönliches Erleben und die Kultur einer Zeit den Blick für bestimmte Gegebenheiten öffnen oder verschließen. Ziel und Ergebnis liegen hier in einer wachsenden Sensibilisierung des Lernenden für künstlerische Mittel und das Verständnis für die Möglichkeit verschiedener Interpretationen des gleichen Textes. (Hombitzer 1986: 992f.)

Wichtig ist Hombitzer in diesem Zusammenhang, dass den Lernenden der Gehalt des Dramas erst in der künstlerischen Gestaltung voll zum Genuss werden kann, denn ïm Miterleben durch Phasen der Erwartung, Überraschung und Modifikation des Gewussten oder Erwarteten klären sich Vorstellungen, wird Formulierung dessen, was bisher 'unformuliert' und unformulierbar war, möglich."(ibid. 994). Hier zeigen sich Berührungspunkte mit Gibsons Ansatz, der die Bedeutung der Aufführungspraxis besonders hervorhebt (vgl. Gibson 1998, 2000). Auch Müller-Schwefe weist auf diesen Aspekt hin:

Wer also - in der Schule oder in privater Lesesituation - ein Drama lesend erschließen will, muss ständig die nicht-verbalen akustischen und visuellen Wirkungsmittel in der Vorstellung, die er sich von der Bühnenrealisation macht, mitvollziehen. (Müller-Schwefe 1986: 731)

Dies gilt nun in besonderem Maße für Shakespeare Dramen, denn sie ënthalten kaum Nebentexte und lassen vor allem in den spärlichen Bühnenanweisungen Hinweise auf die nicht-verbalen Wirkungselemente vermissen"(Müller-Schwefe 1986: 731). Diese Tatsache stellt nicht nur eine Herausforderung für jeden Film- und Bühnenregisseur dar. Sie enthält auch ein nicht zu unterschätzendes didaktisches Potenzial für den Shakespeare-Unterricht mit Blick auf szenische Darstellungsformen und den Einsatz von Verfilmungen.

Einen etwas anderen Ansatz in Bezug auf Shakespeare-Verfilmungen verfolgt Ott (2000), denn sie argumentiert funktional ausgehend von der ohnehin schon 
großen Bedeutung des Mediums Film für Jugendliche und fragt hier nach Möglichkeiten, Romeo für die MTV-Generationërfahrbar zu machen.

Auf der Grundlage von Baz Luhrmanns Romeo-and-Juliet-Verfilmung zeigt Ott Möglichkeiten der Schüleraktivierung, die u.a. auch durch das moderne setting des Films (Bandenkrieg an einer Tankstelle in den USA) begünstigt werden. Die Beibehaltung der Originalsprache sieht sie dabei nicht als Schwierigkeit, Bondern als Chance (...), denn die Schüler waren so gezwungen, sich wesentlich mehr auf die Bildersprache zu konzentrieren und ihre Botschaften zu entschlüsseln."(Ott 2000: 22).

Ein Bewusstsein für die Wirkung, die mit der Visualisierung eines Dramentextes einhergeht, stützt sich dabei allerdings nicht allein auf das Entschlüsseln und Interpretieren filmischer Codes. Wenngleich die Vermittlung filmischer Subtexte immer auch durch einen bewussten Einsatz von Einstellungs- und Darstellungsgrößen sowie Kameraperspektiven erzielt wird, so klammert man hier bisweilen einen wichtigen Aspekt aus, den Hickethier aufzeigt:

Erzählen ist in den audiovisuellen Medien zumeist verbunden mit dem Darstellen (Hervorhebung C.L.). Darin liegt die Besonderheit des Audiovisuellen, dass es durch die inzwischen schon scheinbar selbstverständliche technische Verbindung von Bild und Ton die Bilder erzählbar macht und damit zugleich das Erzählen visualisiert. (Hickethier 2001: 25)

Hier manifestiert sich eine Schnittstelle zwischen film- und dramendidaktischen Ansätzen, denn die Visualisierung eines Dramentextes - aber auch eines Romans - im Film erfordert nicht nur filmtechnische Arrangements, sondern ist ganz unmittelbar an die schauspielerische Darstellung der Figuren gebunden. Unterstützt durch eine Vielzahl filmästhetischer Gestaltungsmöglichkeiten, wird die Inszenierung eines Hamlet bei Michael Almereyda mit Ethan Hawke eben anders gelöst als bei Laurence Olivier, Kenneth Branagh oder Franco Zeffirellis Mel Gibson-Hamlet. Dass hier die Regisseure und Schauspieler einen immer wieder neuen Blick auf den Hamlet-Text werfen (müssen), kann als großes Potenzial zur Schüleraktivierung gesehen und entsprechend genutzt werden. Szenische Interpretationen und vergleichende Filmanalysen können hier wertvolle methodische Ansätze bieten (vgl. Lütge 2007).

\section{Dramendidaktische Überlegungen}

Mit dem aufführungsorientierten Ansatz ist in den letzten Jahren die szenische Umsetzung im Shakespeare-Unterricht stärker in den Blickpunkt gerückt. Gibsons Script Approach (1998) hat daran maßgeblichen Anteil. Hier wird ein aktiver Umgang mit Shakespeares Dramen propagiert, der berücksichtigt, dass diese primär für Aufführungszwecke geschrieben wurden. Gibson selbst erläutert seinen Script Approach folgendermaßen:

(...) treating Shakespeare as a script (and calling it so) suggests a provisionality and incompleteness that anticipates and requires imaginative, dramatic enactment for completion. (Gibson 1998: 7) 
Dabei wird die Unvollständigkeit des Skriptes betont, das einer dramatischen Umsetzung bedarf, um den Text durch die Kraft der Inszenierung zum Leben $\mathrm{zu}$ erwecken. Am Beispiel Hamlets zeigt Laurenz Volkmann auf, wie eine "performance-orientierteMethodik der Schüleraktivierung aussehen kann. In Anlehnung an Lee Strasbergs method acting schlägt er dabei ein performanceorientiertes Rollenspiel vor, das die Schüler für kurze Zeit mit der darzustellenden Hamletfigur verschmelzen lässt (vgl. Volkmann 1997: 33) und dabei auch der Entwicklung eines kritischen Methodenbewusstseins zuträglich ist. Auch Antor (1997: 6) verweist auf die Notwendigkeit, "die Vielstimmigkeit des shakespeareschen Textes deutlich herauszuarbeiten, wobei die somit erarbeitete Dialogizität den Rezeptionsprozess selbst erfassen und dadurch schüleraktivierend wirken soll."

Für Antor besteht ein wichtiges Lernziel jedes fortgeschrittenen Literaturunterrichts in der Bewusstmachung der "Bedingtheit verschiedener Interpretationen, auch der eigenen"(ibid. 6). Im Rahmen des Lernziels einer umfassenden hermeneutischen Kompetenz, die auch langfristig die Befähigung zu einer kritischemanzipierten Auseinandersetzung mit Texten anstrebt, sollte sowohl die Beschäftigung mit dem Shakespearetext als auch mit seinen Interpretationen stattfinden (vgl. ibid. 6). Die Begegnung der Lerner mit Volkmanns multiplen Hamlets"(1997: 33) hat in diesem Zusammenhang eine wichtige Funktion zu erfüllen:

In immer neuen Experimenten in der Theaterwelt geht es nicht primär darum, den Barden aus Stratford als Dead White Male Poet vom Podest der Hochkultur zu stoßen; vielmehr wird angestrebt, in intensiven Inszenierungsexperimenten festgefahrene Rezeptionsmuster und erstarrte Aufführungskonventionen aufzubrechen sowie Berührungsängste mit dem Klassiker abzubauen. (Volkmann 1997: 33)

In der unterrichtspraktischen Umsetzung des aufführungsorientierten Ansatzes lassen sich hier zwei Linien verfolgen, wie Schmidt (2004: 55) aufzeigt:

- Der Besuch von Shakespeare-Aufführungen und/oder der kontrastive Einsatz von Filmen wird begleitend, vor oder nach der Dramenlektüre in den Unterricht eingebettet. In beiden Fällen steht das Interesse an der Begegnung mit einer Inszenierung im Vordergrund, und zwar mit dem Ziel, Einsichten zu vermitteln, wie und mit welcher Wirkung Shakespeare-Texte auf der Bühne und im Film umgesetzt werden."(ibid. 45; Hervorhebung C.L.)

- Die szenische Umsetzung einzelner Szenen durch die Schüler im Rahmen einer prozess- und handlungsorientierten Vorgehensweise kann in einer sehr individuellen Auseinandersetzung mit einzelnen Aspekten des Dramas zu einer intensiven Beschäftigung mit dem Text und Überlegungen der Aufführungspraxis führen.

Nun lassen sich beide Richtungen aber fruchtbar aufeinander beziehen, da sie ein verbindendes Moment haben, nämlich das Interesse an der Inszenierung und 
Aufführungspraxis. Dabei profitiert ein Unterricht, der Formen szenischer Darstellung zur Erarbeitung von Shakespeare-Dramen nutzt, von den Einsichten in die darstellerische Umsetzung im Film und auf der Bühne. Einblicke in die Möglichkeiten einer Bühnenaufführung oder filmischen Umsetzung können Anregungen liefern für eigene darstellerische Ideen der Schüler. Umgekehrt sensibilisiert die eigene Erfahrung im szenischen Interpretieren für die Schauspielkunst auf der Bühne oder im Film, weil hier - wenn auch nur in Ansätzen - erfahrbar wird, wie herausfordernd die körperliche und stimmliche Realisierung einer Dramenszene im Detail ist.

Formen szenischen Interpretierens ermöglichen zudem durch die Aktivierung aller Sinne und die Einbeziehung des ganzen Körpers einen ganzheitlichen $\mathrm{Zu}$ gang zum Lernen, wie man ihn gerade in den fortgeschrittenen gymnasialen Jahrgangsstufen selten findet. Als eine Art entdeckenden Lernens ermöglicht szenisches Interpretieren dabei eine Literaturbetrachtung, die nicht nur analytische Fähigkeiten anspricht, denn es ërfordert und fördert gleichzeitig soziale, sinnliche, motorische, sprachliche und emanzipierte Verhaltensweisen"(Bergmann/Kroth 2002: 7). Hier können Erfahrungen mit der eigenen Individualität gemacht und Einsichten in Möglichkeiten und Beschränkungen darstellerischen Ausdrucks gewonnen werden. Wenn der Text auf diese Weise nicht nur imaginativ, sondern körperlich erfahrbar wird, ergibt sich so ein reichhaltiges Ërprobungsfeld für Perspektivenübernahme und das Ausloten der eigenen Identität"(Spinner 2001: 5). In einem an den Prinzipien der Didaktik des Fremdverstehens orientierten Unterricht spielt die Fähigkeit zur Perspektivenübernahme eine besondere Rolle. Ganzheitliche, kreative Zugänge zum Fremdsprachenunterricht, die Kompetenzen wie Empathie, Toleranz, Perspektivenübernahme und Interaktionsfähigkeit betonen, sind in besonderem Maße geeignet, die Voraussetzung zu einem vertieften Verständnis für fremdkulturelle Aspekte zu schaffen (vgl. Bergmann/Kroth 2002: 7).

Wie Volkmann ausführt, kann ein auf affektive Identifikation und ganzheitliche Wahrnehmung abzielender Ansatz nicht nur Einblicke in Fragestellungen einer Drameninszenierung bieten und in schauspielerische Grundprobleme einführen, sondern auch durch die Rollenerfahrung zu einem besseren Textverständnis der Schüler führen (vgl. Volkmann 1997: 33). Die kreative Spannung im Verhältnis von Text und performance kann so einerseits für künstlerische Zwecke einer ëchten"Bühnenaufführung genutzt werden, denn ëntzieht sich die Bedeutung eines Textes nach Aussage vieler Kritiker der Fixierung, so konkretisiert sich in Momenten der performance flüchtige Sinnhaftigkeit."(ibid.)

Doch mehr als das: Durch Perspektivenwechsel in einem performance-orientierten Shakespeare-Unterricht können Lerner dazu gebracht werden, ihre Erwartungen und Sehgewohnheiten zu befragen; sie müssen das "Wirklichkeitsmodell des Dramas, des Regisseurs und des Filmbesuchers miteinander in Verbindung bringen"(Hombitzer 1986: 994), was eine sehr anspruchsvolle und komplexe Aufgabe ist. In diesem Zusammenhang zeigt Antor auf, dass das Lernziel einer performanceorientierten Unterrichtseineit zu Shakespeare die Entwicklung einer kritischen Theaterkompetenz ist,

die den Schüler nicht nur dazu befähigen soll, bei der Lektüre des Shakes- 


\begin{abstract}
pearetextes dessen konkrete Bühnendimension mitzureflektieren und diesen damit in seiner ursprünglichen Funktion als play text zu verstehen, sondern ihn auch in die Lage versetzen soll, individuelle Konkretisierungen des Dramas auf der Bühne oder im Film informiert, aber unabhängig von den Gemeinplätzen des Besprechungswesens aus seiner eigenen Warte begründet zu evaluieren. (Antor 1997: 9)
\end{abstract}

Antor betont hier stärker die rezeptive Ausprägung, regt aber auch an, Schülern die Gelegenheit zu bieten, Szenen aus Shakespeare selbst zu inszenieren und anschließend in gemeinsamer Diskussion zu reflektieren. Dies kann nun nicht unvermittelt geschehen, sondern bedarf einer behutsamen Heranführung, insbesondere bei Lerngruppen, die wenig Erfahrung mit Formen szenischer Umsetzung haben. Ohnehin geht es beim szenischen Interpretieren im Englischunterricht nicht darum, dass Schüler fremdsprachliches Theater spielen. Vielmehr soll die Darstellung von Beziehungen einzelner Charaktere zueinander bzw. der Ausdruck von Stimmungen und Atmosphäre in den Blick genommen werden (Bergmann/Kroth 2002:7).

Nun darf das didaktische Potenzial performance-orientierter Zugänge nicht darüber hinweg täuschen, dass bei einseitiger Ausrichtung im Klassenzimmer der eigentliche Text aus dem Blick geraten kann (vgl. Schmidt 2004: 25). Ebenso warnt auch Antor vor den Folgen einer Vernachlässigung des Shakespearetextes zugunsten seiner Interpretationen:

Der Schüler muss nach wie vor lernen, seine eigenen Interpretationen wie die der Kritiker vor ihm an den Möglichkeiten des konkreten Textes zu messen anstatt lediglich nur 'um den Text herumzureden', ohne sich direkt auf diesen zu beziehen."(Antor 1997: 7)

Dies gilt in gleichem Maße für szenische Darstellungen von Shakespearetexten, die ihren Ausgangs- und Referenzpunkt am Text haben sollten. Durch die Kontrastierung der szenischen Gestaltung mit filmischen Umsetzungen kann aber ein Bezugsrahmen gesetzt werden, der eine Arbeit am und mit dem Text besonders fördert.

\title{
4 Methodische Möglichkeiten: Schüleraktivierung mit Drama und Film
}

Ein Shakespeare-Unterricht, der sich an den neueren performance studies und an film studies orientiert, ermöglicht einen besonders schülernahen Unterricht, der "die Lektüre eines verstaubten Klassikers zum lebendigen Erlebnis für den Lerner werden lassen kann"(Antor 1997: 9). Schüleraktivierung birgt dabei ein hohes Motivationspotenzial und kann die deskbound approaches ersetzen, die eher zu passiver Rezeption führen, sei es nun von Texten, Filmen oder Bühnenaufführungen.

Führt man sich aber vor Augen, dass bei älteren Schülern häufig mit einer ëntwicklungsbedingten Scheu vor dem szenischen Darstellenßu rechnen ist (Schmidt 
2004: 52), dann wird die Notwendigkeit einer behutsamen Heranführung an die Thematik besonders deutlich. Wie Antor (vgl. 1997: 7) betont, darf das Motivationspotenzial eines solchen schüleraktivierenden Ansatzes nicht durch die Frustration der Überforderung des Lerners wieder zerstört werden und bedarf daher genauer methodischer Vorbereitung. Ganz grundsätzlich stellt sich zudem die Frage, ob man auch eher zurückhaltende, introvertierte Schüler in performanceorientierte Verfahren integrieren kann, bzw. wie dies methodisch möglich ist.

Neben einer behutsamen Hinführung ist dafür auf Seiten der Lehrkraft auch die Akzeptanz vonnöten, dass nicht alle Schüler auf die gleiche Art und Weise am Unterricht partizipieren (müssen). Im Folgenden möchte ich einige konkrete Anregungen geben, wie Elemente einer performance-orientierten Vorgehensweise mit dem Einsatz von Shakespeare-Verfilmungen kombiniert werden können.

\subsection{Aktiv durch Schweigen ? - Behutsame Annäherungen}

Was zunächst ungewöhnlich klingen mag, ist bei genauerer Betrachtung durchaus plausibel: die Aktivierung von Schülern über das Schweigen. Antor erklärt dies folgendermaßen:

auch das Schweigen oder das Verstummen von Charakteren wird durch den Ansatz der performance studies erst als dramatisches Instrument erkannt und kann als wichtige Interpretationshilfe dienen. Wie verhalten sich beispielsweise schweigende oder verstummte Personen auf der Bühne? Welche Bedeutung hat dies für ihr Verhältnis zu anderen Charakteren. (Antor 1997: 8)

Wichtig erscheint mir in diesem Zusammenhang auch der Hinweis auf die Funktion, die Pausen insbesondere in den langen Shakespeare-Monologen zu erfüllen haben, denn sie strukturieren und gliedern den Text und tragen dazu bei, Stimmungen zu erzeugen. Bereits Ausgesprochenes mag so nachklingen, die Erwartung auf noch zu Sagendes gesteigert werden. Gut dosiert und geschickt gesetzt, wird das Schweigen als gestaltendes Element genutzt und kann unter dramendidaktischen Gesichtspunkten in den Unterricht integriert werden.

Hier bieten sich vielfältige methodische Möglichkeiten an, die auch weniger extrovertierten Schülern Möglichkeiten bieten, sich in einen performance-orientierten Shakespeare-Unterricht einzubringen:

- Erst einzeln, dann in der Gruppe strukturieren die Schüler den Text, indem sie Entscheidungen über Pausen treffen. Sie überlegen gemeinsam, an welchen Stellen Hamlet verstummen sollte, und begründen, warum dies sinnvoll ist. Verstummt er abrupt, oder wird er vorher langsamer, spricht er danach laut oder leise weiter? Ist es z.B. sinnvoll, in Hamlets To be or not to beMonolog vor und/oder nach der Zeile Äy, there's the rub"(Hamlet, III, $1,65)$ deutliche Pausen zu setzen? Ebenso lässt sich der Schluss des Monologs diskutieren: "And thus the native hue of resolution / Is sicklied o'er with the pale cast of thought / And enterprises of great pitch and moment / With this regard their currents turn awry / And lose the name of action... 
Soft you now / The fair Ophelia." (Hamlet III, 1, 84-89). Wie wird die Pause zwischen "and lose the name of action" und "soft you now" gestaltet? Wird sie lange gehalten? Bildet sie eine Brücke zur Anrede Ophelias? Dies probieren die Schüler selbst aus, testen dabei die Wirkung und diskutieren das Ergebnis.

- Auch aus der Frage, wie sich Hamlet in der Pause verhält, lassen sich schüleraktivierende Verfahren entwickeln. Welche Gestik und Mimik ist hier möglich? In welcher Körperhaltung und Position im Raum agiert der Schauspieler? Das lässt sich in der Gruppe nicht nur thematisieren, sondern auch visualisieren.

- Die vorangegangenen Überlegungen leiten ebenfalls in Gruppenarbeit zu der Frage über, wie ein Regisseur im Film Pausen darstellen kann, an welchen Stellen Großaufnahmen, Schnitte oder Positionswechsel erfolgen.

- Mit zunächst nur einer Filmversion vergleicht die Gruppe ihre eigenen Vorschläge für die Gestaltung der Pausen des Monologs. Lässt man die vier Verfilmungen von Olivier, Zeffirelli, Branagh und Almereyda zwischen den Gruppen austauschen, können die Kontraste in Bezug auf Pausengestaltung gezielt erarbeitet und anschließend diskutiert werden. ${ }^{4}$ Die Reduzierung der Komplexität der dramatischen Handlung auf ein Phänomen wie das der Pause kann so in der Kombination eigener szenischer Darstellung und filmischer Umsetzung aktiv erarbeitet werden.

Antor verweist zudem auf die sogenannten ßpeaking silences", die in verschiedenen Shakespearetexten ähnliche Fragen aufwerfen (vgl. Antor 1997: 8). Als Beispiele nennt er u.a. das Schweigen Antonios am Ende von Twelfth Night und die Gerichtsszene von Measure for Measure, in der Isabella nach dem Heiratsantrag des Herzogs schweigt. Wie Antor betont, können Schüler hier gut dazu angeleitet werden, Antworten zu finden, die dann gegenüber Mitschülern verteidigt werden können und für die schlüssige Vorschläge zur dramatischen Umsetzung auf der Bühne gemacht werden können. Auf diese Weise "durchläuft der Schüler in der Auseinandersetzung mit Shakespeare einen aktiven Prozess gestaltenden und kritischen Lernens, der ihn als emanzipierten Rezipienten zum Zuge kommen lässt."(ibid. 8)

\subsection{Körpersprachliches Agieren: Nonverbale Textbegegnungen}

Will man Shakespearetexte mit Schülern szenisch darstellen, erfolgt recht häufig eine frühzeitige Rollenverteilung oder -zuweisung, die dann zu einem immer wieder zu beobachtenden Phänomen führt: Die beteiligten Schüler stehen mehr oder

\footnotetext{
${ }^{4}$ Mit Blick auf die Pause nach Änd lose the name of actionlässt sich die unterschiedliche Realisierung in den Filmen diskutieren. Laurence Olivier hat die im Originaltext folgende nunnery scene vorgezogen, bei Michael Almereyda folgt sie an späterer Stelle, Kenneth Branagh dagegen hält die Reihenfolge des Shakespearetextes bei.
} 
weniger bewegungslos an prominenter Stelle im Klassenraum in der Gruppe beieinander, eine Hand vielleicht in der Hosentasche versenkt, mit der anderen den Text haltend, von dem beim Deklamieren kaum der Blick gehoben wird. Je nach Temperament wird zuweilen eine theatralische Geste eingeflochten, die Stimme vielleicht erhoben in Erwartung amüsierter Reaktionen aus der Menge der zuschauenden Klassenkameraden. Kaum je wird körpersprachlich agiert, wird der Raum schreitend durchmessen, zeigen Positionswechsel Veränderungen der Atmosphäre oder Stimmung an. Im Grunde genommen lesen Schüler hier stehend Texte lediglich $a b$. Tatsächlich wären sie auch überfordert, sollten sie ohne Vorbereitung sprachlich agierend Shakespearetexte weitgehend frei vortragen, um dabei noch Raum für darstellerische Variationen zu finden, deren Umsetzung in der Gruppe dann noch einmal eine ganz eigene Dynamik entfaltet.

'Filmbilder' können im Sinne einer pre-acting activity genutzt werden, um Eindrücke zu sammeln, wie Shakespeare-Dramen darstellerisch umgesetzt werden. Soll ein Monolog erarbeitet werden, so bietet sich eine tonlose Präsentation an, die die Aufmerksamkeit auf die nonverbalen Aspekte lenkt. Es ist ratsam, einen ähnlich langen, in jedem Fall aber einen anderen als den anschließend zu bearbeitenden Monolog auszuwählen, um eine spätere bloße Nachahmung der Schüler zu vermeiden. Ausgehend von der Erfahrung mit einer filmischen Variante können dann unterschiedliche Verfahren genutzt werden, um den Text körperlich erfahrbar zu machen. Dazu eignen sich einerseits pantomimische Darstellungen, die in Kleingruppen erarbeitet und ggf. auch in der größeren Gruppe präsentiert werden. Eine andere Variante mag sich als besonders motivationsfördernd erweisen. Dazu stellt eine Schülergruppe gemeinsam eine von drei vorher erarbeiteten Textabschnitten, z.B. aus größeren Monologen dar. Der Rest der Klasse muss nun erraten, um welchen Textabschnitt es sich handelt. Trotz rein körpersprachlicher Darstellung bleibt der Text dabei präsent; und alle Schüler werden aktiv am Unterrichtsgeschehen beteiligt.

Auch das 'Sammeln räumlicher Erfahrungen' ist wichtig, um ein Gefühl für die vielfältigen Möglichkeiten der Schauspielkunst zu gewinnen. Welchen Bewegungsradius hat ein Charakter, wie häufig und wie weit schreitet er oder sie den Raum $a b$, wann und warum wird am Platz verharrt? Die Vorführung einer Dialogszene aus einer Shakespeare-Verfilmung kann im Rahmen einer while-viewing activity genutzt werden, um Bewegungsdiagramme anzufertigen. Andere Textstellen können von den Schülern dann in ähnlicher Form bearbeitet werden. Ideen, die hier in der Gruppe entwickelt werden, können dann ausprobiert und gemeinsam diskutiert werden.

Das 'Bauen von Standbildern' (freeze frames) gehört zu den beliebten nonverbalen Einstiegen ins szenische Interpretieren (vgl. Bergmann/Kroth 2002: 7). So kann nach Abschluss einer Szene oder eines Aktes durch die Lernenden ein Standbild erstellt werden, das den Stand der Beziehungen im Drama widerspiegelt. Auf diese Weise können Schüler über das dramatische Potenzial der folgenden Szenen, sich entwickelnde Konflikte oder Dilemmata der Protagonisten spekulieren. Bergmann illustriert eine konkrete Vorgehensweise für A Midsummer Night's Dream und Romeo and Juliet, indem sie aufzeigt, wie mögliche role cards zu diesen Wer- 
ken aussehen könnten, um das Beziehungsgeflecht der Charaktere darzustellen (Bergmann 2002: 29 f.). Eine solche Aktivität kann gut aufgegriffen werden, um überzuleiten auf die Präsentation eines sogenannten freeze frames, d.h. eines Standbildes einer Filmszene. Dabei ergeben sich vielfältige Möglichkeiten des Vergleichs zwischen der eigenen und der filmischen Umsetzung. Auch umgekehrt können freeze frames aus Shakespeareverfilmungen dazu anleiten, eigene Standbilder zu präsentieren. Das mag sowohl in einem möglichst ähnlichen Nachstellenöder aber einer bewusst sehr stark veränderten Variante der Szene resultieren. Auf jeden Fall lassen sich auch hier Formen szenischer Inszenierung mit einer Verfilmung sehr gut verbinden.

\subsection{Verbale Improvisationen: Stimmen und Stimmungen}

Die Variationsmöglichkeiten im künstlerischen Ausdruck ergeben sich u.a. auch durch den gezielten Einsatz der Stimme. Um für sehr unterschiedliche darstellerische Realisierungen eines Henry $V$ - einmal von Laurence Olivier und einmal von Kenneth Branagh - vorbereitet zu sein, bedarf es nicht nur der Beachtung filmtechnischer Details, wie oben schon ausgeführt wurde. Gerade an SchauspielerRegisseuren wie Olivier und Branagh lassen sich auch Beobachtungen hinsichtlich des sprachlichen Gestus machen, die von großer Bedeutung für den künstlerischen Gesamteindruck sind.

Wenn Schüler in einfachen Übungen an verbales Improvisieren herangeführt werden, um mit ihrer eigenen Stimme SStimmungenßu erzeugen, dann findet hier nicht nur eine in dramendidaktischer Hinsicht wünschenswerte Form der Schüleraktivierung statt, sondern zugleich auch eine Sensibilisierung für eine künstlerische Ausdrucksform. Als einen möglichen Einstieg schlägt Bergmann (2002: 28) eine Aktivität vor, bei der Schüler durch den Raum schreiten und sich gegenseitig begrüßen mit Originalformulierungen (z.B. How, now...; Good mistress,...; Welcome hither,...; Hail to thee...). Denkbar sind auch kurze Abschnitte aus großen Monologen, auch und gerade wenn diese sehr bekannt sind wie To be or not to be"(vgl. Lütge 2007). Um die Schüler dazu zu bringen, nun mit der Stimme unterschiedliche Stimmungen zu erzeugen, ist es hilfreich, wenn die Lehrkraft zusätzliche Anweisungen gibt, die bei der verbalen Realisierung beachtet werden sollen. So rät Bergmann (2002: 28), Textzeilen in folgender Weise realisieren zu lassen:

- as if you say 'hello' in a friendly manner

- as if you were talking to your lover

- as if you were passing a secret

- as if you were telling a joke

- in a threatening manner

- in a cold, controlled manner 
- as if you were flattering the person you meet

Auch Variationen in der Lautstärke und Geschwindigkeit des Vortrags können von Schülern gut ausgelotet werden, wenn sie sich im Schutz einer Gruppenaktivität durch den Raum bewegen und dabei ihre Zeile deklamieren. Eine gleichzeitige Bewegung ist in der Tat empfehlenswert, weil durch die aufrechte Haltung eine freiere Atmung unterstützt wird und gleichzeitige körperliche Bewegungsabläufe den Mut zur verbalen Improvisation eher fördern als ein Verharren am Tisch. Eher introvertierte Schüler finden hier Gelegenheit, sich stimmlich auszuprobieren, ohne sich vor einer Gruppe exponieren zu müssen. Viele methodische Abwandlungen sind denkbar, etwa ein dialogisches Deklamieren von zwei Schülern, die sich im Raum begegnen. Wenn unterschiedliche Realisierungen einer Zeile wie to be or not to be"buchstäblich im Raum stehen, bietet sich eine vergleichende Betrachtung der Umsetzung in unterschiedlichen Hamlet-Verfilmungen an. Mit anderen Augen - und auch Ohren - nehmen stimmlich erprobte Schüler die Unterschiede wahr zwischen Laurence Oliviers, Mel Gibsons, Kenneth Branaghs und Ethan Hawkes Rezitationen. Dies kann als Einstieg in die Behandlung des Monologs, des ganzen Hamlet oder in entsprechend abgewandelter Form als Hinführung an andere Shakespeare-Dramen erfolgen.

\subsection{Gruppenerfahrungen: Gemeinschaftliche Texterkundungen}

Die gemeinsame Erfahrung bei der szenischen Erkundung eines dramatischen Textes kann gruppendynamisch eine wichtige Rolle spielen. Gruppenrezitationen bringen Spontaneität in den Unterrichtsablauf, denn sie liefern häufig überraschende, wenig geplante bzw. planbare Sichten auf den Text. Zum Einstieg in die Behandlung eines Dramas oder eines bestimmten Aspektes eignet sich eine Übung, die in der dramendidaktischen Literatur als 'taking lines across the circle' bekannt ist. Bergmann (2002: 28) beschreibt, wie bei diesem Verfahren ein Monolog oder eine Textzeile immer wieder neu entstehen kann. Zu diesem Zweck stellen sich die Lehrkraft und die Schüler in einem Kreis auf. Abwechselnd geht nun eine Person durch den Kreis auf einen Mitschüler zu und spricht dabei eine Textzeile, die zuvor auf einer Karteikarte notiert wurde. Wenn die Textzeile so "durch den Kreis getragen wurde", tauschen die beiden Schüler ihren Platz. Variationen in der Gestik, Mimik und Bewegung ermöglichen die Erprobung unterschiedlicher Wirkungen der gleichen Textzeile. Auf diese Weise kann beobachtet und ausprobiert werden, wie mehrere Versionen des gleichen Texts von verschiedenen Schülern realisiert werden. Auch kürzere Abschnitte aus Monologen, die bereits gut bekannt sind, können auf diese Weise umgesetzt werden. Eine solche Vorgehensweise kann auch nach der Präsentation der entsprechenden Textstelle aus einer oder mehreren Shakespeare-Verfilmungen gewählt werden, um eine Kontrastierung mit der eigenen Tätigkeit zu ermöglichen.

Gemeinschaftliche Texterkundungen bieten sich auch in der Kombination mit verbalen und nonverbalen Elementen an. So können Textabschnitte in einer Gruppe durch mehrere Schüler pantomimisch dargestellt werden, während ein oder zwei Schüler als Sprecher den dazugehörigen Text deklamieren. Sie machen so 
die Erfahrung, dass das Zusammenwirken vieler für den Gesamteindruck einer Szene von großer Relevanz ist und dass hier in einem vielstimmigen Chor Bedeutung erzeugt wird.

\subsection{Szenarien entwerfen: Visualisieren und Inszenieren}

Wie Hickethier ausführt, liegt die Besonderheit des Audiovisuellen darin, dass es "durch die scheinbar selbstverständliche technische Verbindung von Bild und Ton die Bilder erzählbar macht und damit zugleich das Erzählen visualisiert."(Hickethier 2001: 25) Der Aspekt der Visualisierung kann gut umgesetzt werden in schüleraktivierende Verfahren, die sich u.a. mit Fragen der Ausgestaltung von Bühnenbildern beschäftigen.

Die Erstellung von Filmskripten zur Inszenierung von Szenen mit einem anschließenden Vergleich mit der Verfilmung ist eine beliebte Methode, um auf analytische und zugleich kreative Art und Weise mit Shakespeare-Texten zu arbeiten. Ein Raster mit vorgegebener Tonspur (vgl. Abb. 1) ermöglicht eine strukturierte Vorgehensweise (vgl. Lütge 2007, Groene/Kieschke 1986).

Tabelle 1: Raster zur Erstellung eines Filmskripts

\begin{tabular}{|l|c|c|c|c|c|}
\hline setting, action & \multicolumn{2}{|c|}{ camera } & \multicolumn{2}{c|}{ soundtrack } \\
\hline & $\begin{array}{c}\text { camera } \\
\text { dis- } \\
\text { tance }\end{array}$ & $\begin{array}{c}\text { camera } \\
\text { angle }\end{array}$ & $\begin{array}{c}\text { camera } \\
\text { move- } \\
\text { ment }\end{array}$ & speech & music/ \\
sound
\end{tabular}

Aber auch freiere Varianten der Visualisierung in eher assoziativer Form sind denkbar, und zwar sowohl in Einzel-, Partner- oder Gruppenarbeit. Hier können Überlegungen zu Requisiten und Kostümen, vorherrschenden Farben oder Lichtverhältnissen, zur musikalischen Untermalung und Raumgestaltung angestellt werden. Skizzen des Bühnenbildes können gemeinsam entworfen und wiederum mit einer filmischen Umsetzung verglichen werden.

Ott beschreibt, wie ihre Schüler anhand von Romeo and Juliet eigene Ideen zur Umsetzung der ersten Filmszene entwickelten (Ott 2000: 23). Eine Möglichkeit besteht in der Ausgestaltung eines Bewegungsdiagramms (s.o.), das in Form einer Skizze mit einfachen Symbolen die Position der Schauspieler und ihrer Bewegungs- und Blickrichtungen verdeutlicht. Dieses Verfahren bietet sich als Gruppenarbeit an, bei der die Szene einerseits gemeinsam besprochen, andererseits aber auch szenisch nachgestellt werden kann. Die Anfertigung von Bewegungsdiagrammen bietet also etwa im Rahmen einer pre-viewing activity die Möglichkeit, in gemeinschaftlicher Arbeit Überlegungen zur Inszenierung einer Textstelle anzustellen. Die Schüler müssen dabei versuchen, den Ablauf zu visualisieren und tun dies häufig mit spontanen Inszenierungen. Die Lehrkraft kann die Schüler ggf. auch ermutigen, die Praktikabilität ihrer Bewegungsdiagramme zu 
überprüfen, indem der Raum durchschritten wird, die anvisierten Positionen eingenommen oder Blickrichtungen geändert werden. Auf diese Weise können Ideen auch verworfen und neu entwickelt werden. Die körperliche Erfahrbarkeit wirkt dabei nicht nur motivierend und schüleraktivierend, sondern bietet Möglichkeiten zur Entwicklung kreativer und ästhetischer Kompetenzen, die der Filmanalyse wiederum zugute kommen.

\subsection{Regie reflektieren: Verfilmungen auf dem Prüfstand}

In einem performance-orientierten Shakespeare-Unterricht, der nach der szenischen Darstellung eine oder mehrere Filmversionen zur Kontrastierung mit einbezieht, können schüleraktivierende Elemente auch in einer abschließenden Reflexionsphase ihren Raum finden. Dazu eignet sich z.B. eine Podiumsdiskussion, bei der Schüler die Rollen der jeweiligen Regisseure, einiger Schauspieler, möglicherweise auch des Kameramanns oder der Requisiteure, Maskenbildner u.ä. übernehmen, während die Schüler im Plenum Diskussionen zu einzelnen Aspekten der filmischen Umsetzung initiieren. Etwa in Form einer Pressekonferenz können sich hier in einer Lerngruppe lebhafte Diskussionen entwickeln, in deren Verlauf die Podiumsmitglieder ihre Entscheidungen zur Umsetzung des Dramentextes in einen Film erläutern. Die Wahrnehmung einer anderen Perspektive ermöglicht dabei häufig eine sehr intensive Auseinandersetzung mit den jeweiligen Positionen, insbesondere wenn sie in einem kritischen Dialog mit dem Schüler-Publikum erfolgen. Bei der Einbeziehung einer Podiumsdiskussion am Beispiel mehrerer Hamlet-Verfilmungen in einem Englisch-Leistungskurs zeigte sich, dass ein hohes Maß an Schüleraktivierung erreicht werden konnte durch die Kontrastierung der Filmversionen von Laurence Olivier, Kenneth Branagh und Michael Almereyda (vgl. Lütge 2007). Wenn Schüler die Rolle eines Regisseurs, Kameramannes oder Kritikers übernehmen, müssen sie analytische und kreativ-produktive Fähigkeiten entwickeln und reflektieren so eine vorhergehende Filmpräsentation aktiv und engagiert. Gleichzeitig schlüpfen sie in eine andere Rolle und können ihre Position in einem anderen Szenario fremdsprachlich agierend erproben.

\section{Schlussbemerkung}

Bei einem über die traditionelle Vorgehensweise eines deskbound approach hinausgehenden Shakespeare-Unterrichts muss keineswegs eine Entscheidung zwischen dem Einsatz von Shakespeare-Verfilmungen einerseits und einer performanceorientierten Methodik andererseits getroffen werden. Vielmehr lassen sich dramenund filmdidaktische Ansätze gut miteinander verbinden im Rahmen eines an den Prinzipien der Handlungsorientierung ausgerichteten Englischunterrichts (vgl. Bach/Timm 2003). Schüleraktivierende Verfahren, die einen Perspektivenwechsel befördern und die Selbstreflexivität der Schüler an der Schnittstelle zwischen einem Dramentext und seiner Verfilmung unterstützen, leisten einen wichtigen Beitrag zur Entwicklung einer umfassenden Sprachhandlungskompetenz. Hier ist 
es wichtig, methodische Möglichkeiten im Blick zu haben, die auch eine behutsame Annäherung an die szenische und filmische Umsetzung ermöglichen und den zuweilen etwas unvermittelten SSprung"vom Text zum Bild abmildern (vgl. Lütge 2007). Formen szenischer Interpretation im Shakespeare-Unterricht haben damit nicht nur eine motivationale Funktion, sondern sie vermögen zwischen den Texten zu vermitteln. Dramentext und filmischer Text werden gemeinsam befragt, eigene Deutungsmuster werden in der interpretierenden Darstellung der Lerner ausgehandelt und kontrastiert. Das schüleraktivierende Potenzial im Zusammenwirken szenischer Interpretationen und Verfilmungen kann so im Shakespeare-Unterricht fruchtbar gemacht werden.

\section{Bibliographie}

Antor, Heinz (1997): Now set thy long-experienc'd wit to school. Neue Shakespeare-Forschung und alternative Literaturdidaktik. In: Der Fremdsprachliche Unterricht Englisch 31/30, 4-9

Bach, Gerhard; Timm, Johannes-Peter (Hg.) (2003): Englischunterricht. 3., vollst. überarb. und verb. Auflage. Tübingen: Francke (UTB)

Bergmann, Birgit (2002): Texts and Tasks. In: Der Fremdsprachliche Unterricht Englisch 36/56, 27-34

Bergmann, Birgit / Kroth, Olivia (2002): Much is the force of heaven-bred poesy. In: Der Fremdsprachliche Unterricht Englisch 36/56, 4-10

Brusch, Wilfried (2002): Discovering Shakespeare: A media-integrated approach. In: Finkbeiner, Claudia (Hg.): Wholeheartedly English: A Life of Learning. Festschrift für Johannes-Peter Timm. Cornelsen: Berlin, 267-280

Duncker, Ludwig; Sander, Wolfgang; Surkamp, Carola (Hg.) (2005):

Perspektivenvielfalt im Unterricht. Stuttgart: Kohlhammer

Hickethier, Knut (2001): Film- und Fernsehanalyse. Stuttgart: Metzler

Hombitzer, Eleonore (1986): Die Shakespeare-Verfilmung im Englischunterricht der gymnasialen Oberstufe. In: Ahrens, Rüdiger (Hg.): William Shakespeare, Didaktisches Handbuch, München: Fink (UTB), Bd. 3, 987-1013

Gibson, Rex (1998): Teaching Shakespeare. Cambridge: Cambridge University Press

Gibson, Rex (2000): Active Methods in Shakespeare Classrooms. In: Der Fremdsprachliche Unterricht Englisch 34/46, 17-21

Groene, Horst; Kieschke, Klaus (1986): Literaturverfilmung im

Englischunterricht der Sekundarstufe II. Frank R. Stocktons 'The Lady or the Tiger?' In: Praxis des neusprachlichen Unterrichts (1), 3-12

Klose, Hartmut (2000): He writes real people. In: Der Fremdsprachliche Unterricht Englisch 34/46, 4-9

Lütge, Christiane (2007): Hamlet goes to Hollywood: vom szenischen Interpretieren zur vergleichenden Filmanalyse im Leistungskurs Englisch. In: 
Ahrens, Rüdiger; Eisenmann, Maria; Merkl, Matthias (Hg.): Moderne Dramendidaktik für den Englischunterricht der Sekundarstufe I und II. Heidelberg: Winter (im Druck)

Müller-Schwefe, Gerhard (1986): Shakespeares Dramen in der Filmkunst. In: Ahrens, Rüdiger (Hg.): William Shakespeare, Didaktisches Handbuch, München: Fink (UTB), Bd. 2, 729-756

Ott, Alexandra (2000): Romeo für die MTV-Generation? In: Der Fremdsprachliche Unterricht Englisch 34/46, 22-26

Roma, Gill (Hg.) (1994): William Shakespeare. Hamlet, Oxford: Oxford University Press

Schmidt, Isolde (2004): Shakespeare im Leistungskurs Englisch. Eine empirische Untersuchung. Frankfurt: Lang

Spinner, Kaspar (2001): Spielszenen im Deutschunterricht. In: Praxis Deutsch 166, 4-9

Surkamp, Carola (2004): Teaching films. Von der Filmanalyse zu handlungsund prozessorientierten Formen der filmischen Textarbeit. In: Der Fremdsprachliche Unterricht Englisch 38, 2-8

Thaler, Engelbert (2001): DVD im Englischunterricht. In: Der Fremdsprachliche Unterricht Englisch 35/53, 40

Volkmann, Laurenz (1997): The ten Hamlets: Eine performance-orientierte Methodik der Schüleraktivierung. In: Der Fremdsprachliche Unterricht Englisch 31/30, 32-35 